\title{
The Koch Monopole: A Small Fractal Antenna
}

\author{
Carles Puente Baliarda, Member, IEEE, Jordi Romeu, Member, IEEE, and Angel Cardama, Member, IEEE
}

\begin{abstract}
Fractal objects have some unique geometrical properties. One of them is the possibility to enclose in a finite area an infinitely long curve. The resulting curve is highly convoluted being nowhere differentiable. One such curve is the Koch curve. In this paper, the behavior the Koch monopole is numerically and experimentally analyzed. The results show that as the number of iterations on the small fractal Koch monopole are increased, the $Q$ of the antenna approaches the fundamental limit for small antennas.
\end{abstract}

Index Terms-Fractals, small antennas.

\section{INTRODUCTION}

$\mathbf{F}$ RACTAL-SHAPED antennas have already been proved to have some unique characteristics that are linked to the geometrical properties of fractals. As it was thoroughly discussed in [1]-[4], the self-similarity property of fractals makes them specially suitable to design multifrequency antennas. In this paper, we present and discuss another interesting property of fractal shapes that has a large potential to increase the performance of antennas. Some fractal shapes have complex, convoluted shapes that can enhance radiation when used as antennas. For instance, some fractal loops can be designed to enclose a finite surface with an arbitrarily large perimeter. Certain monopoles can be designed to have an arbitrarily large length, although they can be constrained to fit a given volume. Therefore, it is possible to design small antennas that occupy the same volume than their Euclidean counterparts, but much longer. This interesting property has been used in the design of Frequency Selective Surfaces (FSS) [5]. In this case, resonant elements that occupy a small volume were built after a fractal shape. The underlying potentiality is to design small and efficient antennas that have a fractal shape. The implications of such designs, as well as how they relate to some well-known antenna restrictions, is discussed in the following sections.

When the size of an antenna is made much smaller than the operating wavelength, it becomes highly inefficient. Its radiation resistance decreases, while, proportionally, the reactive energy stored in the antenna neighborhood rapidly increases. Both phenomena make small antennas difficult to match to the feeding circuit, and when matched, they display a high $Q$, i.e., a very narrow bandwidth. The potentiality of fractal shapes for improving the efficiency of common small antennas has been suggested by Cohen in [6]-[8]. Based on a numerical method of moments analysis, he observed that fractal Minkowski loops presented a low resonant frequency, relative to their electric size.

Manuscript received September 16, 1999; revised April 6, 2000. This work was supported by CICYT and the European Commission by Grant FEDER 2FD97-0135.

The authors are with the Department of Signal Theory and Communications, Telecommunication Engineering School, Universitat Politecnica de Catalunya, Spain.

Publisher Item Identifier S 0018-926X(00)07718-8.
In this paper, the behavior of the Koch monopole is presented. While some preliminary results were discussed by the authors in [9], this paper provides an indepth insight to the behavior of the antenna and its relation to the fundamental limits of antennas. In Section II, the fundamental limitations of small antennas are reviewed. In Section III, the fractal monopole Koch antenna is described and its input parameters are shown. Both numerical and experimental results show that as the number of iterations in the monopole increase, the $Q$ of the antenna approaches the fundamental limit for small antennas. The current distribution along the antenna and the possible existence of a limit in its performance is discussed in Section IV. Section V shows the radiation pattern of the antenna. It is shown that as the frequency increases, the asymmetry in the antenna geometry manifests in the radiation pattern. Finally, in Section VI, the possible use of the Koch monopole as a multiband antenna is discussed.

\section{Fundamental Limitations on SMall ANTENNAS}

This section is devoted to review some aspects regarding small antennas. Several authors have extensively worked upon the fundamental limits of small antennas [10]-[17], Chu's and Wheeler's work being among the ones that have most influenced further investigations on such a topic. An antenna is said to be small when it can be enclosed into a radiansphere, i.e., a sphere with radius $a=\lambda / 2 \pi$. For such antennas, Chu established a fundamental limitation on their $Q$ given by [17]:

$$
Q=\frac{1}{k^{3} a^{3}}+\frac{1}{k a}
$$

which establishes the lower, fundamental limit of the $Q$ factor that can be achieved by a linearly polarized antenna. Several authors have reported similar results, some of them leading to a slightly different expression for (1) [11]. Recently, McLean [14] has reviewed some of these concepts and has shown that the fundamental limit upon circularly polarized antennas is of the order of one half of that of the linearly polarized ones.

It is important to state that this limit is established regardless of the antenna current distribution inside the sphere. Actually, the current distribution inside the sphere is not uniquely determined by the field distribution outside the sphere [17], so several current distributions can lead to the same $Q$ factor. In practice, such a fundamental limit has not ever been reached, being the Goubau antenna [12] with $Q=1.5 \cdot Q_{\min }$ one of the lowest $Q$ reported antennas. Achieving a low $Q$ antenna basically depends on how efficiently it uses the available volume inside the radiansphere.

It must be stressed that Chu's definition of the $Q$ factor is a general concept that applies even when the antenna is not resonant. Since the $Q$ definition is valid for a resonant system, it is 
implicitly assumed that the extra amount of unbalanced reactive energy of a nonresonant antenna is externally compensated by a proper inductor or capacitor at the input terminals. Therefore, depending on the nature of the predominating energy, the out of resonance $Q$ factor has to be defined as [18]

$$
\begin{array}{ll}
Q=\omega \frac{2 W_{e}}{P_{r}} & W_{e}>W_{m} \\
Q=\omega \frac{2 W_{m}}{P_{r}} & W_{m}>W_{e}
\end{array}
$$

where $W_{m}$ and $W_{e}$ are the stored and magnetic energies and $P_{r}$ is the average radiated power. Of course, both definitions are equivalent if the antenna is self-resonant, which is not usual for small antennas. Prior to Chu's work, Wheeler introduced the Power Factor (PF) concept to characterize small antennas [10], [13]. He assumed that any small reactive antenna could be reduced to an equivalent first-order network when operating at low frequencies. Such a network would be either a serial RC network or a parallel RL one depending on whether electric or magnetic stored reactive energy predominates at low frequencies. He defined the PF for such networks as

$$
\begin{aligned}
\mathrm{PF}_{e} & =\frac{G}{B}=\frac{G}{\omega C} & W_{e}>W_{m} \\
\mathrm{PF}_{m} & =\frac{R}{X}=\frac{R}{\omega L} & W_{m}>W_{e}
\end{aligned}
$$

with $B$ and $X$ being the radiation susceptance and reactance, and $G$ and $R$ the radiation conductance and resistance, respectively. Actually, such definition can be reinterpreted as $\mathrm{PF} \approx$ $1 / Q$, provided that only the radiation resistance or conductance is taken into account, and that the contribution of only one of the reactive components of the antenna input impedance is significant to the overall antenna performance. Also, he stated that the lowest achievable PF is that of a spherical coil filling the volume inside the radiansphere, that is

$$
\mathrm{PF}=\left(\frac{2 \pi a}{\lambda}\right)^{3}=k^{3} a^{3}
$$

which is in agreement with the limit established in (1), provided that the radius of the sphere enclosing the antenna is much smaller than the operating wavelength.

\section{The SMALl Koch MonOPOLE}

First thought of as a candidate to become a simple multiband antenna, the Koch monopole is an effective example to illustrate that fractals can improve some features of common Euclidean shapes. Being ideally a nonrectifiable curve, its length grows as $(4 / 3)^{n}$ at each iteration, the Koch curve has neither a piecewise continuous derivative. Actually, it is nowhere differentiable, which means that its shape is highly rough and uneven. Thus, it appears as a good candidate for becoming an efficient radiator.

\section{A. Antenna Description}

The classical Koch curve construction algorithm is very well known. An Iterative Function System (IFS) algorithm can be applied to generate the succession of curves that converge to

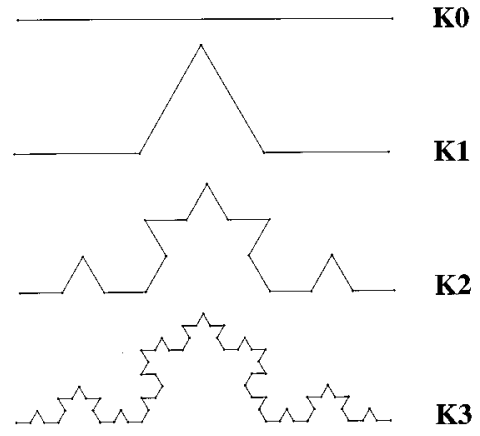

Fig. 1. Different iteration stages of the Koch curve. One stage is obtained by applying the affine transformation of (9) to the previous one.

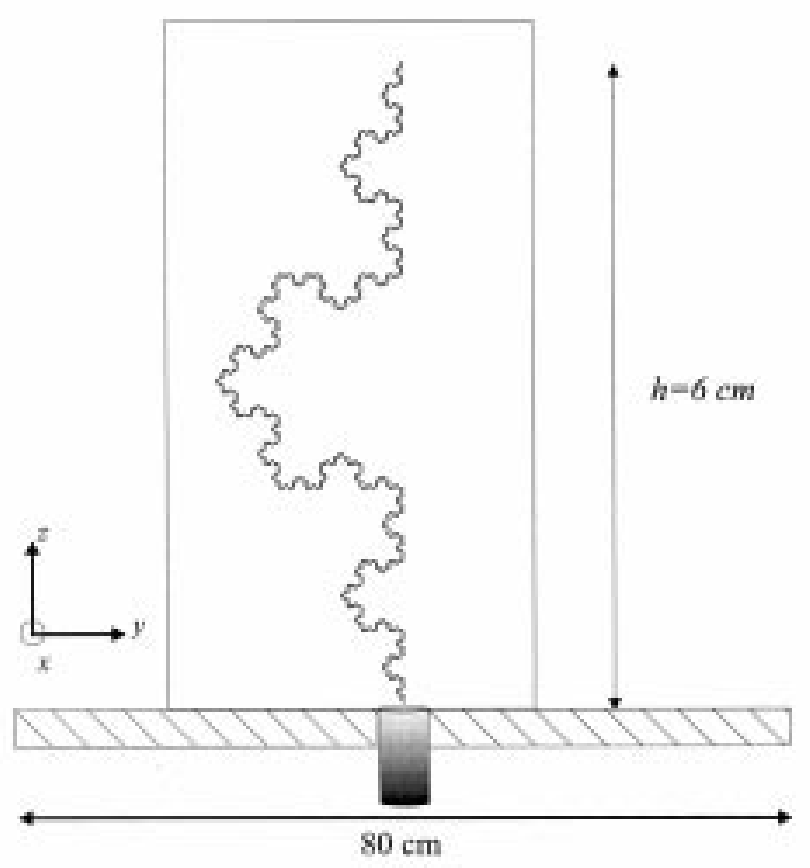

Fig. 2. Five-iteration Koch monopole over the ground plane used in the gathering of experimental data. The whole length of the element is $l=h \cdot(4 / 3)^{5}=25.3 \mathrm{~cm}$.

the ideal fractal shape. These IFSs are used to construct certain fractals. Rather than a rigorous mathematical description that can be found elsewhere [19], only the relevant points will be outlined. An IFS is defined by a set of affine transformations. An affine transformation in the plane $\omega: \mathbb{R}^{2} \rightarrow \mathbb{R}^{2}$ can be written as

$$
\omega\left(\begin{array}{l}
x_{1} \\
x_{2}
\end{array}\right)=\left(\begin{array}{ll}
a & b \\
c & d
\end{array}\right)\left(\begin{array}{l}
x_{1} \\
x_{2}
\end{array}\right)+\left(\begin{array}{l}
e \\
f
\end{array}\right)=A x+t
$$

where $x_{1}$ and $x_{2}$ are the coordinates of point $x$. For short, and through this paper, the affine transformations will be written as $\omega=[a, b, c, d, e, f]$. The matrix $A$ can always be written as

$$
A=\left(\begin{array}{rr}
r_{1} \cos \theta_{1} & -r_{2} \sin \theta_{2} \\
r_{1} \sin \theta_{1} & r_{2} \cos \theta_{2}
\end{array}\right)
$$

In the special case where $r_{1}=r_{2}=r, 0<r<1$, and $\theta_{1}=\theta_{2}$, the transformation is a contractive similarity where $r$ 


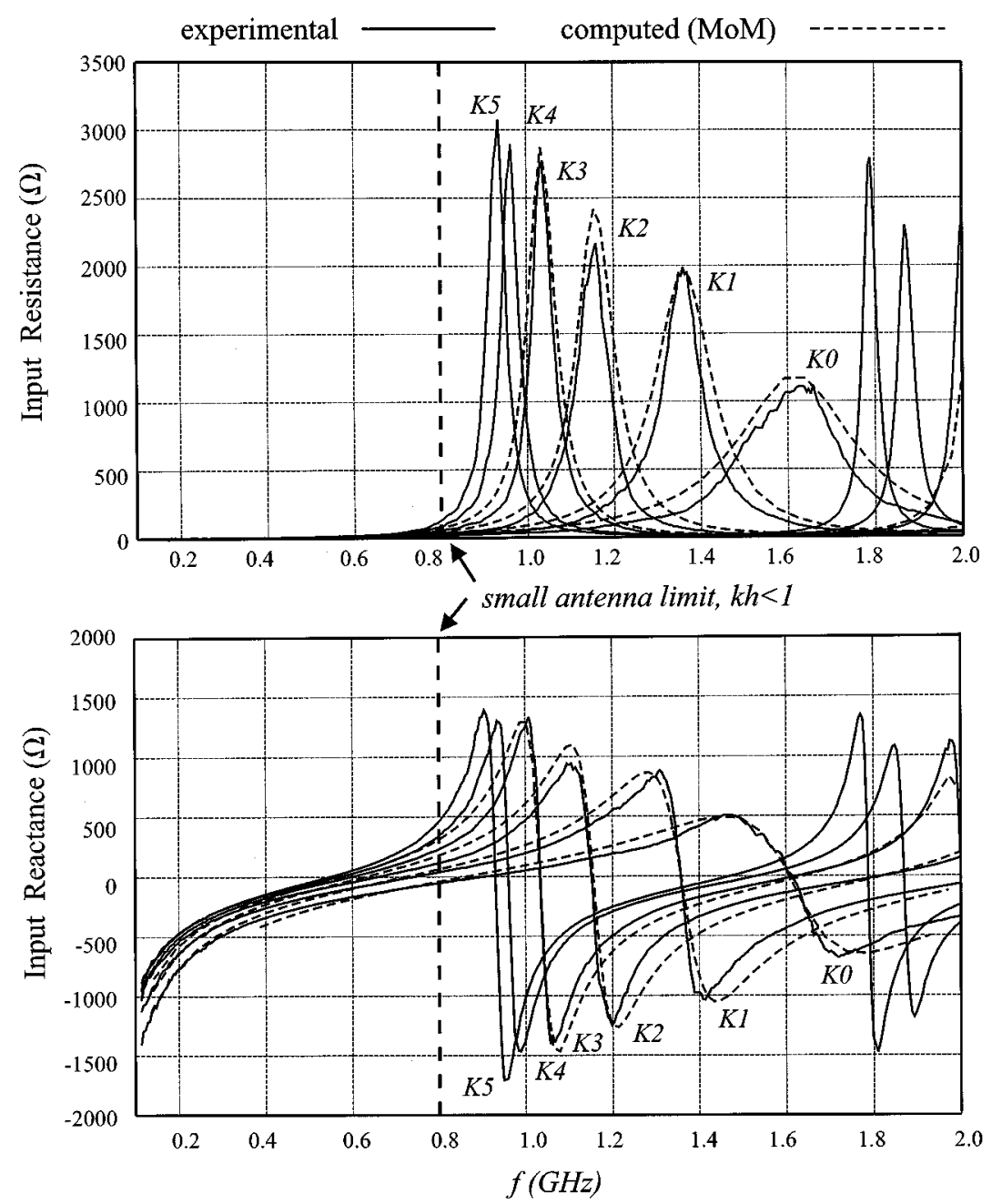

Fig. 3. Input impedance of the different fractal Koch monopoles. Numerically simulated data are in good agreement with measurements. As the number of iterations in the structure increases the resonant frequency diminishes.

is the scale factor and $\theta$ is the rotation angle. Note that a similarity is an affine transformation where shear transformations are not allowed, therefore in similarity, transformation angles are preserved [20]. The column matrix $t$ is just a translation on the plane. Following the IFS scheme, the Koch curve can be defined from the following set of affine transformation:

$$
\begin{aligned}
\omega_{1}= & {[1 / 3,0,0,1 / 3,0,0] } \\
\omega_{2}= & {[1 / 3, \cos 60,-1 / 3 \sin 60,1 / 3 \cos 60,} \\
& 1 / 3 \cos 60,1 / 3,0] \\
\omega_{3}= & {[1 / 3 \cos 60,1 / 3 \sin 60,-1 / 3 \sin 60,} \\
& 1 / 3 \cos 60,1 / 2,1 / 2 \sqrt{3}] \\
\omega_{4}= & {[1 / 3,0,0,1 / 3,2 / 3,0] . }
\end{aligned}
$$

These transformations are scalings by a factor of one-third and rotations by $0,60,-60,0^{\circ}$, respectively. Fig. 1 shows the construction process of the Koch curve. The first element of the series is a straight segment and is called $\mathrm{K} 0$. It can be proved that the starting element is irrelevant to converge to the fractal shape [19]; however, when analyzing the fractal antenna behavior, it looks especially interesting to compare it with that of the closest Euclidean version, i.e., a straight monopole. We will name such a straight monopole as $\mathrm{K} 0$ (the zeroth iteration of the fractal construction), while the remaining objects of the iterations will be referred to as $\mathrm{K} 1, \mathrm{~K} 2, \ldots, \mathrm{K} N$. The next iteration $\mathrm{K} 1$, is obtained by applying the four similarity transformations of (9) to K0. The next elements are obtained iteratively. The fractal shape is obtained as the limit element after infinite iterations. Although the fractal shape might look too convoluted to be of practical application, the first iterations can be easily printed over a dielectric substrate using standard printed circuit techniques. The fabrication complexity in this case is exactly the same for the Euclidean antennas and for almost all fractal ones. Since one is interested in examining the low-frequency behavior, a high-performance (low-loss) microwave dielectric substrate is no longer required. Up to five iterations of the fractal succession were constructed, all of them of the same overall height and using the same substrate. The six antennas (K0 ... K5) where mounted over an $80 \times 80-\mathrm{cm}$ ground plane and measured over 
a $0.1-2 \mathrm{GHz}$ frequency range. The antennas were also numerically analyzed using the frequency-domain method of moments technique. Fig. 2 shows the five-iteration version of the Koch monopole (i.e., K5), which has an overall height $h=6 \mathrm{~cm}$, but a whole length of $l=h \cdot(4 / 3)^{5}=25.3 \mathrm{~cm}$.

\section{B. Input Parameters}

The input resistance and reactance of the five measured Koch monopoles, together with the same parameters for a linear monopole (K0) are shown in Fig. 3. All antennas have the same height $h=6 \mathrm{~cm}$, but of course a different length $l$. The method of moments data corresponding to the Euclidean antenna (K0) and the first three iterations of the fractal one (K1, K2, K3) are also shown for comparison. The copper-etched wire had approximately a rectangular cross section of width $w=200 \mu \mathrm{m}$ and thickness $t=35 \mu \mathrm{m}$, except for the K5 model where the wire width was reduced to $w=150 \mu \mathrm{m}$. To get the best match between experimental and numerical data, an equivalent radius of $a=120 \mu \mathrm{m}$ was considered following the equivalent radius approach outlined in [21]. It is apparent that a good match between numerical and experimental data is obtained.

Some interesting conclusions can be derived from the input parameters plot. First of all, in the low-frequency region, the input resistance increases with the number of iterations when comparing the six characteristics at a given frequency. While the linear monopole input resistance becomes very small below the first resonance $(0.9 \mathrm{GHz})$, the $\mathrm{K} 5$ model is about its input resistance maximum value. Fig. 4 shows the drive resistance increase with growing fractal iterations for a fixed frequency (the first monopole resonant frequency has been chosen in this case). Analogously, the input-reactance plot evinces that resonant frequencies are consistently shifted toward the lower frequency region at each fractal iteration. In particular, even though all the Koch models can be considered small antennas $(k h=1)$ for $f<0.8 \mathrm{GHz}$, they are self-resonant, i.e., they have a vanishing input reactance without the need of an external compensating reactive element. The longest antenna (K5) reaches its second resonance at about the same frequency where the linear dipole has its first resonance.

Also, an equivalent input resistance due to ohmic losses was computed at each frequency by integrating the square of the current distribution over the whole antenna length. The skin effect was also taken into account thereby assuming an equivalent cylindrical wire cross section as described in [21]. Fig. 5 shows the evolution of the drive-resistance radiation and the ohmic losses considering an antenna made of copper. As expected, the results show an increment in the antenna ohmic resistance when the number of iterations is increased. Fig. 6 shows that the ohmic efficiency of the antenna is slightly reduced at each iteration; however, the ohmic efficiency is above 0.9 even in the small antenna region.

\section{The Quality Factor}

It has been shown that the fractal Koch antenna improves some features of a classical linear monopole when operating as a small antenna. Namely, resonant frequencies are shifted toward the longer wavelengths at each fractal growth iteration, making

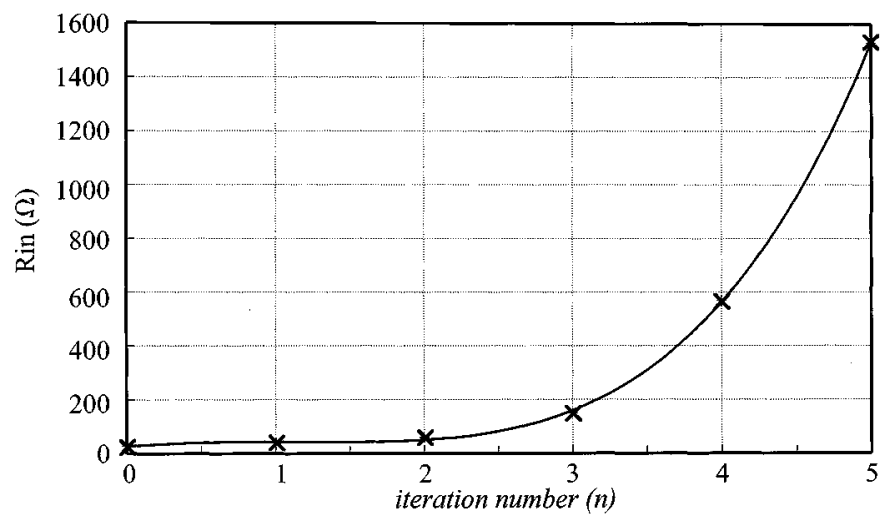

Fig. 4. Input resistance (lossless) for the different fractal Koch monopoles. The frequency is the resonant frequency of the $\mathrm{K} 0$ monopole.

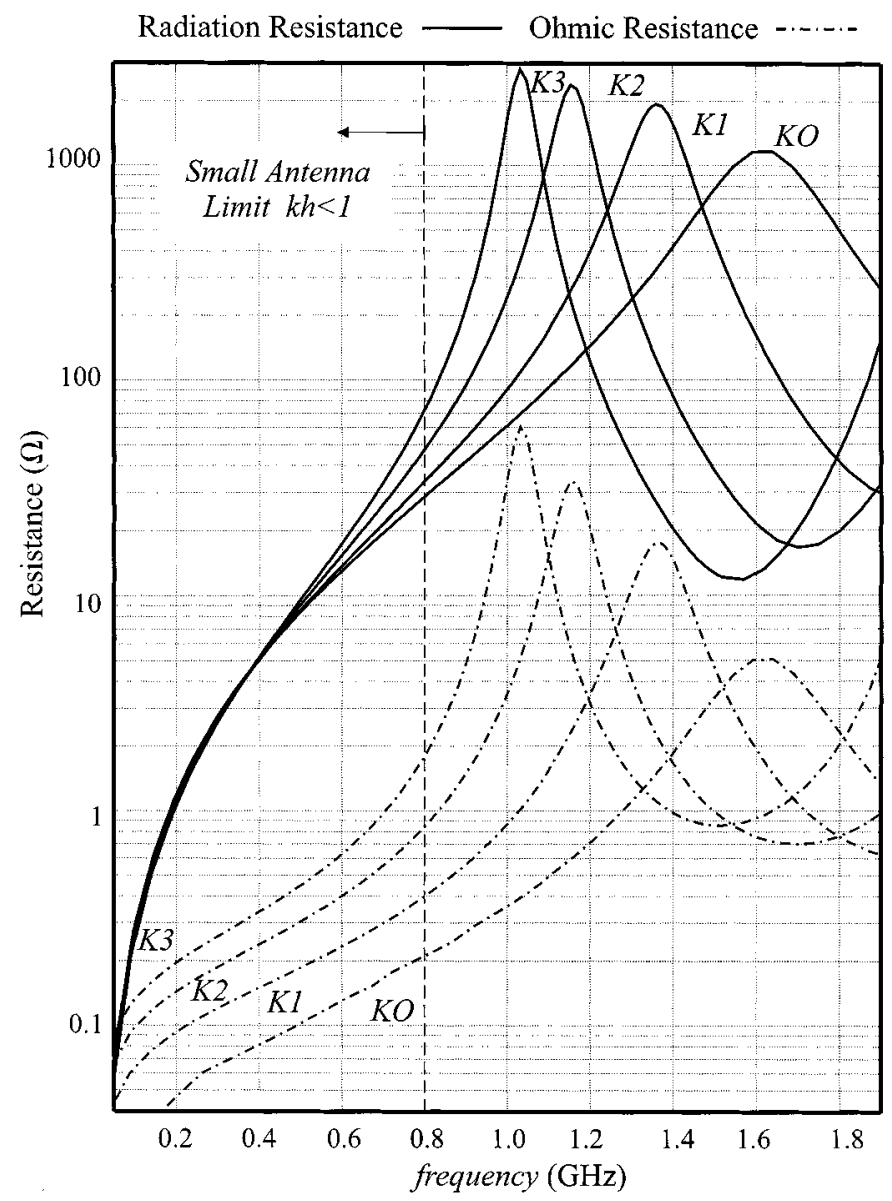

Fig. 5. Computed radiation and ohmic resistance. As the number of iterations increases, so does the antenna length and its ohmic resistance.

the antenna to be resonant even below the small antenna limit. Such a frequency shift makes the input resistance appear consistently larger in the fractal case than in the linear monopole one. However, one must take into account that not only the input resistance is raised, but also that the input reactance is increased. Actually, a figure of merit of the small antenna is its $Q$ factor, which can be loosely estimated as the input reactance $\left(X_{\text {in }}\right)$ to radiation resistance $\left(R_{r}\right)$ ratio. 


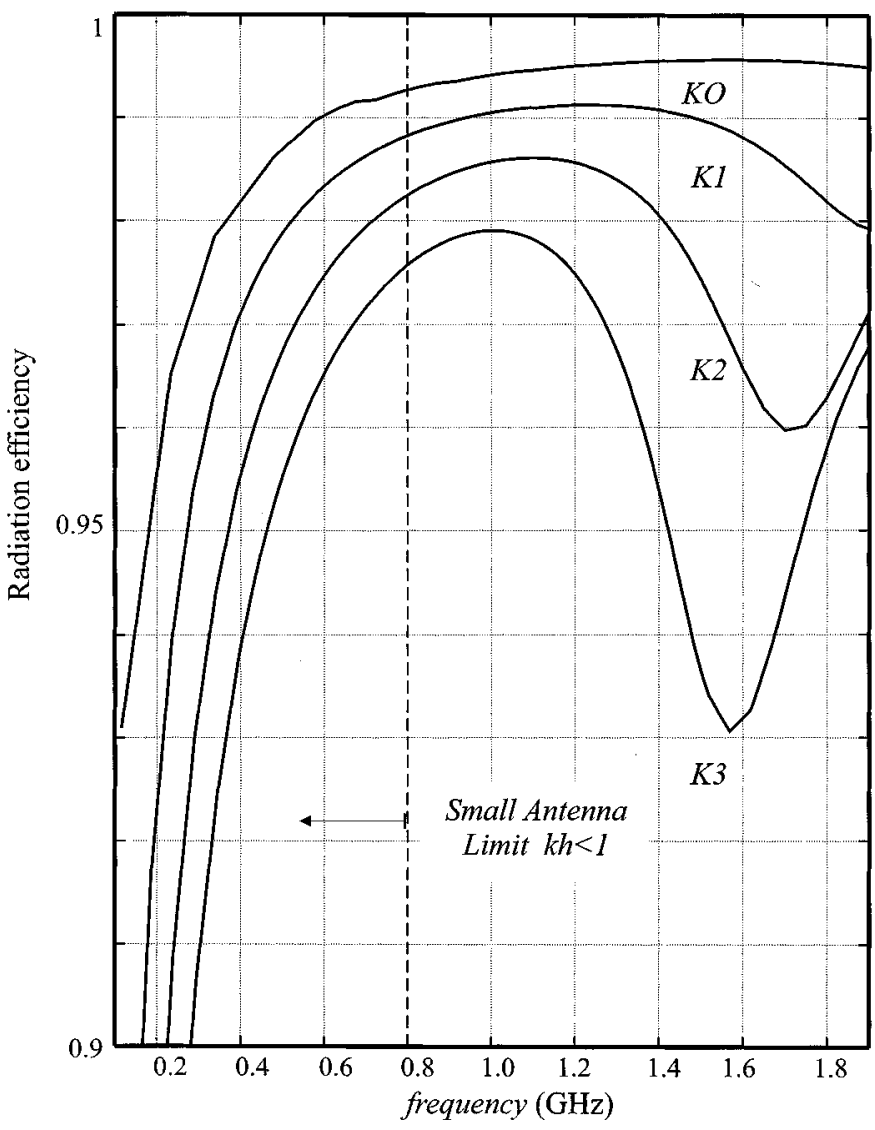

Fig. 6. Efficiency of the Koch monopoles. At each new iteration there is a small reduction on the efficiency due to ohmic losses. The conductivity of copper has been considered in the computations.

The $Q$ factor of the Koch antennas has been computed from both experimental and numerical data. The $Q$ factor, rather than the Wheeler's PF [10], has been chosen to check the antenna performance because the latter might lead to some wrong conclusions in this case. Both parameters are equivalent at the very low-frequency range when the antenna is operated far from resonance. At such a low-frequency region, the $Q$ can be computed from the input impedance data as

$$
Q \approx \frac{1}{\mathrm{PF}} \approx \frac{X_{\mathrm{in}}}{R_{r}}
$$

In such a very low-frequency range, the Wheeler cap method [10], [15], can be used to estimate the antenna ohmic resistance and subtract it from the input resistance to evaluate the true antenna radiation resistance. However, even though the measured Koch antennas can be considered small, neither the Wheeler cap method can be applied, nor its PF computation is straightforward. Since the fractal monopoles are resonant, the contribution of the inductive component to the input impedance extends well beyond the small antenna limit. If one directly applied (10), one would get the wrong conclusion that a zero $Q$ (and an infinite PF) were achieved at resonance. Also, the Wheeler cap method might lead to a wrong radiation resistance measurement since the metallic cap would slightly shift the antenna resonant fre- quencies where fast variations on the input parameters are obtained.

To properly evaluate the antenna $Q$, one must find a proper way to estimate either the average stored electric or magnetic energy and apply the definitions in (2) and (3). The average stored electric and magnetic energies of a lossless one-terminal microwave network can be related to the input reactance $\left(X_{\mathrm{in}}\right)$ and susceptance $\left(B_{\text {in }}\right)$ as

$$
\begin{gathered}
W_{e}=\frac{|I|^{2}}{8}\left(\frac{d X_{\text {in }}}{d \omega}-\frac{X_{\text {in }}}{\omega}\right)=\frac{|V|^{2}}{8}\left(\frac{d B_{\text {in }}}{d \omega}+\frac{B_{\text {in }}}{\omega}\right) \\
W_{m}=\frac{|I|^{2}}{8}\left(\frac{d X_{\text {in }}}{d \omega}+\frac{X_{\text {in }}}{\omega}\right)=\frac{|V|^{2}}{8}\left(\frac{d B_{\text {in }}}{d \omega}-\frac{B_{\text {in }}}{\omega}\right)
\end{gathered}
$$

where $I$ and $V$ are the input terminals current and voltage, respectively. Since the power dissipated by the antenna is

$$
P_{L}=\frac{1}{2}|I|^{2} R_{\mathrm{in}}=\frac{1}{2}|V|^{2} G_{\mathrm{in}}
$$

the $Q$ factor, as defined in (2) and (3), can be computed as

$$
\begin{array}{ll}
Q=\frac{\omega}{2 R_{\mathrm{in}}}\left(\frac{d X_{\mathrm{in}}}{d \omega}-\frac{X_{\mathrm{in}}}{\omega}\right) & W_{e}>W_{m} \\
Q=\frac{\omega}{2 R_{\mathrm{in}}}\left(\frac{d X_{\mathrm{in}}}{d \omega}+\frac{X_{\mathrm{in}}}{\omega}\right) & W_{m}>W_{e}
\end{array}
$$

or equivalently as

$$
Q=\frac{\omega}{2 R_{\text {in }}}\left(\frac{d X_{\text {in }}}{d \omega}+\left|\frac{X_{\text {in }}}{\omega}\right|\right) .
$$

One must be reminded that (11) and (12) only strictly apply to one-port lossless network, but they become a good approximation for low-loss high- $Q$ networks, which is the case of small antennas. Actually, the definition in (15) is the same Chu uses in his paper [17] to derive the antenna $Q$ fundamental limit. Equation (16) has been applied to compute the $Q$ factor over the low-frequency range for the Euclidean and Koch fractal antennas (Fig. 7). Both experimental and numerical data have been used. In the latter case, ideal lossless Koch antennas have been considered to evaluate the antenna $Q$, that is, only considering power dissipation due to radiation. This has been done because the experimental data includes the ohmic resistance that lowers the overall $Q$, which might lead us to the wrong conclusion that the $Q$ reduction was only due to an increase of the ohmic losses. The plot in Fig. 7 clearly shows that the fractal antenna not only presents a lower resonant frequency and a larger radiation resistance, but it also improves the $Q$ factor of the linear monopole. In a loose sense, such $Q$ can be interpreted as the inverse of the fractional bandwidth, which means that the fractal antenna features a broader bandwidth than the Euclidean one. Up to 1.6bandwidth improvement is obtained when comparing the ideal lossless monopole and $\mathrm{K} 3$ antenna, while up to a 2.25-bandwidth enhancement is obtained when comparing the monopole with the K5 antenna, which, however, has the contribution of larger ohmic losses. Anyway, it is clear that the $Q$ factor is reduced at each fractal growth iteration and that, the larger the number of iterations, the closer the $Q$ to the fundamental limit (the fundamental limit expressed in (1) is also shown for com- 


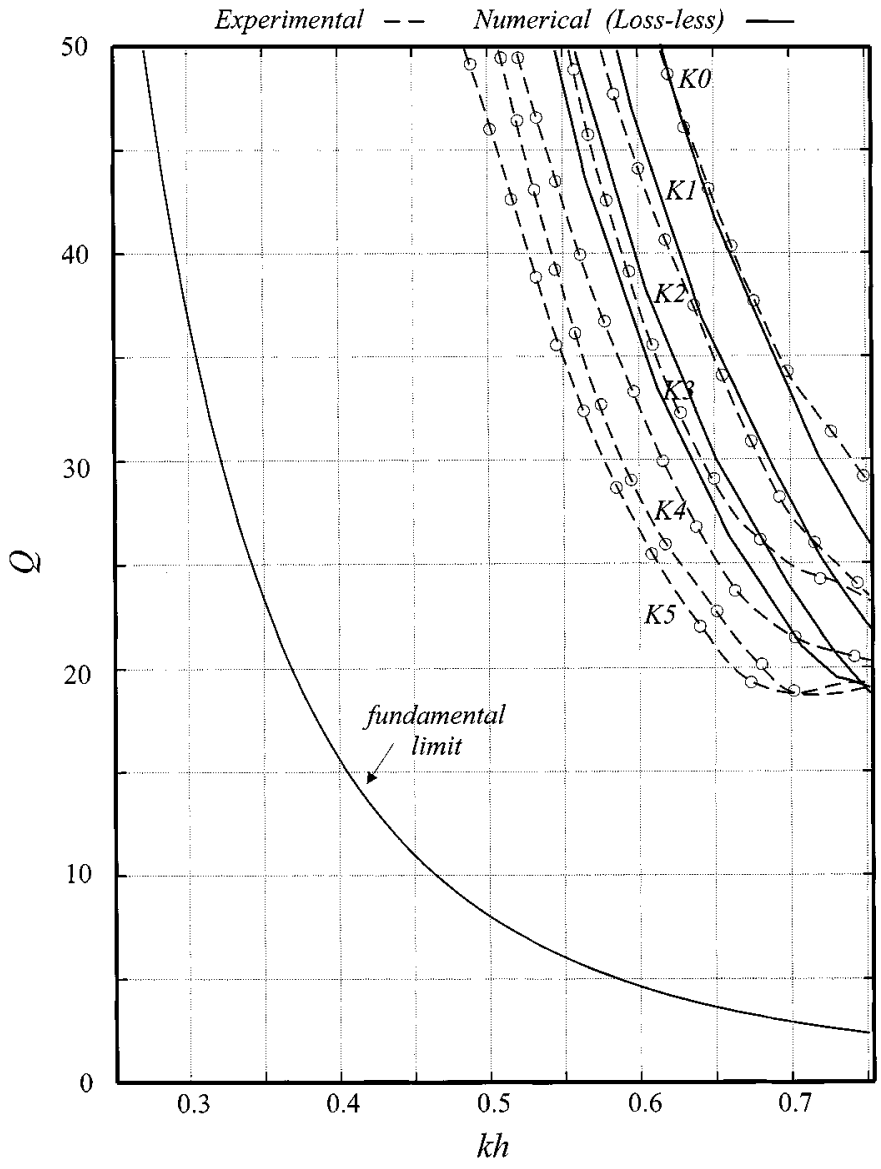

Fig. 7. $Q$ of the fractal Koch monopoles for different levels of iteration. As the number of iterations increases the $Q$ approaches the fundamental limit of (1).

parison). It is commonly understood that an antenna $Q$ factor depends on how efficiently it uses the available volume inside the radiansphere. As stated by Hansen in [11], being the linear dipole a one-dimensional (1-D) object $(D=1)$, it inefficiently exploits such a radiansphere volume. Thus, it is not surprising that a fractal curve, featuring a fractal dimension $D>1(D=$ 1.262 for the Koch monopole), can become a more efficient small antenna. Actually, fractal dimension is commonly loosely interpreted as a measure of the space-filling properties of the fractal object. Therefore, one should conclude again that there exists a relation between the fractal geometric properties and the electromagnetic behavior of the antenna, and that such properties can be readily employed to design useful antennas that might improve some features of common Euclidean ones.

\section{Current Distribution}

The peculiar behavior of the Koch monopole must be linked to its geometrical shape. It is apparent that even though all models have the same height, they perform as longer antennas than what would be predicted from their height alone. It seems that the electrical current propagates along the whole wire length despite its shape such that the longer the whole wire, the lower the resonant frequencies, regardless of antenna size (height).
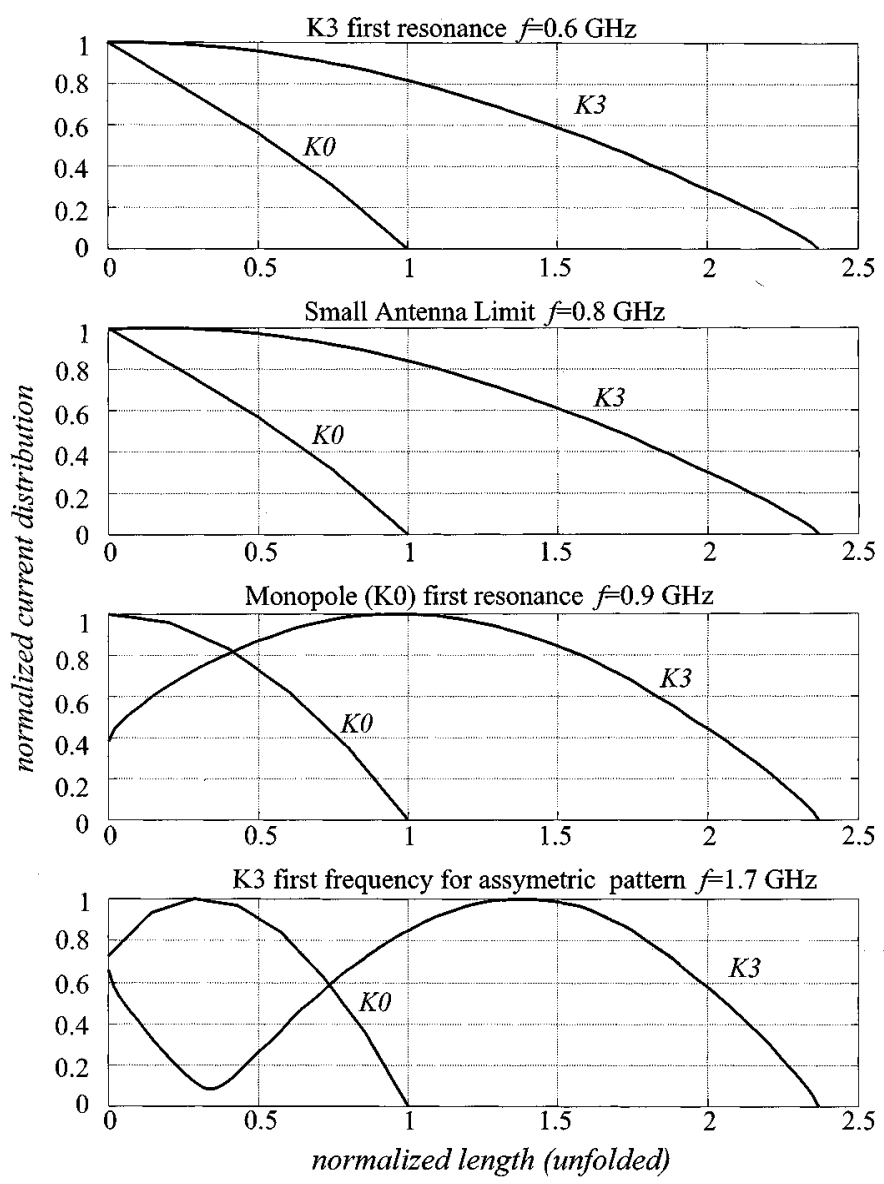

Fig. 8. Current distribution on the K3 monopole.

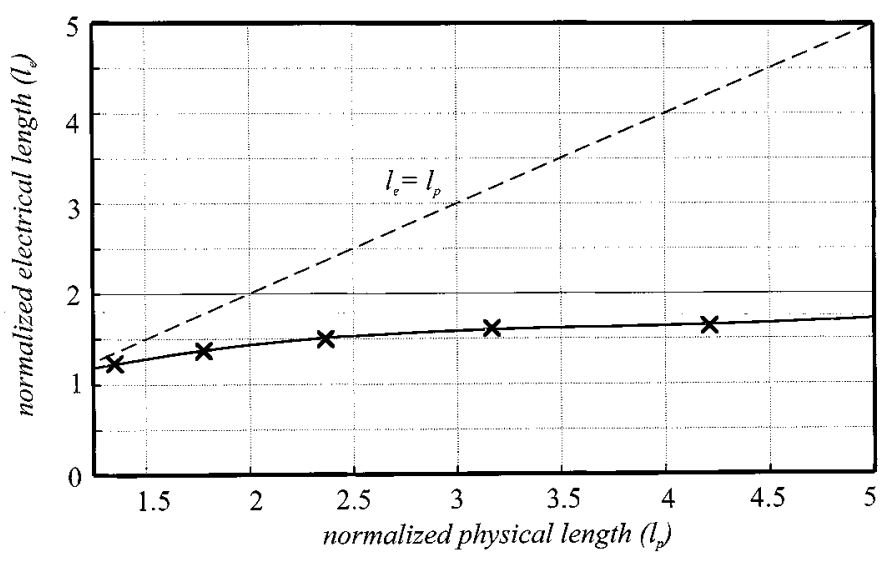

Fig. 9. Performance of the fractal Koch monopole as the number of iterations increase. The electrical length of the antenna increases at a smaller rate that the physical length.

Fig. 8 shows the current distribution along the K3 monopole obtained from the method of moments analysis. The length of the $\mathrm{K} 3$ monopole has been normalized to the length of the straight $\mathrm{K} 0$ monopole $(l=6 \mathrm{~cm})$. Several frequencies of interest are shown to better understand the behavior of the antenna. For comparison, the current distribution for the $\mathrm{K} 0$ monopole is shown for the same frequency. At the first resonance of the $\mathrm{K} 3$ monopole, its length is 

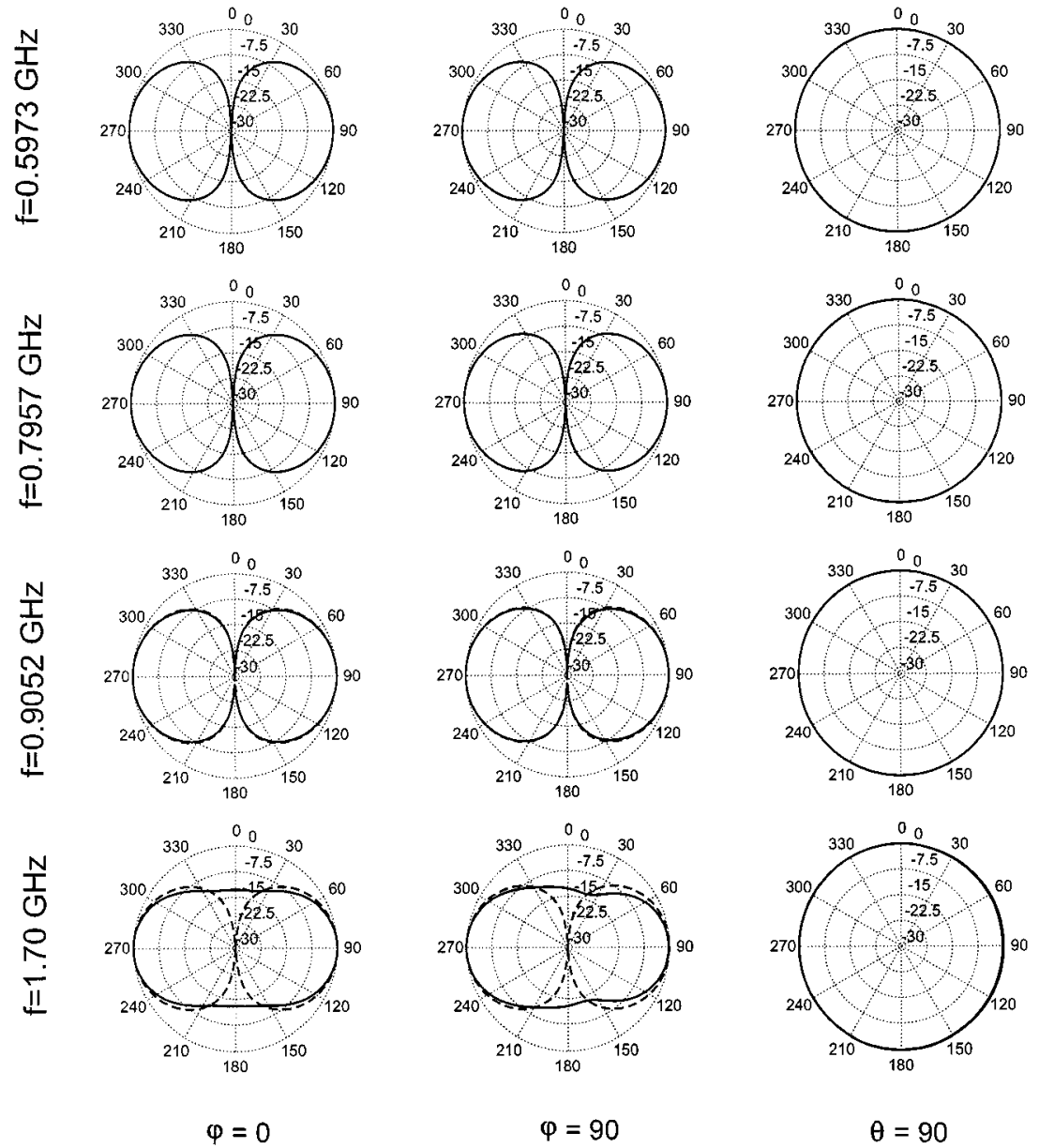

Fig. 10. Computed radiation pattern of the $\mathrm{K} 3$ antenna. The dashed line shows for comparison the pattern of the K0 antenna. For a reference of the coordinate system, see Fig. 2.

$l / \lambda=0.28$, and the current distribution along the $\mathrm{K} 3$ monopole is very similar to the one of a quarter-wave length monopole. At the small antenna limit $(k h=1)$ the current distribution on the $\mathrm{K} 0$ monopole is, as expected, triangular. At the same frequency, the $\mathrm{K} 3$ monopole being 2.37 times longer, has a sinusoidal current distribution. At the frequency of resonance of the $\mathrm{K} 0$ monopole, the $\mathrm{K} 3$ monopole has a length of $0.43 \lambda$, and its current distribution approaches the one of a half-wave length monopole. Finally, the current distribution is shown for the case K3 monopole, which has a length of $0.97 \lambda$. At that frequency, as will shown in the next section, the pattern of the monopole becomes asymmetrical.

The current distribution along the Koch antenna suggests that an arbitrarily small resonant antenna could be obtained by increasing the number of fractal iterations as much as desired, so the length of the monopole approaches a quarter-wave length. Nevertheless, a detailed observation of the results shows a different picture. To gain some insight on the behavior of the Koch monopole, the following two parameters are defined. First, we define the normalized physical antenna length $\left(l_{p}\right)$ as the total length of the antenna normalized to its height, that is

$$
l_{p}=(4 / 3)^{n}
$$

where $n$ is the order of the iteration. Second, we define the normalized electrical length $l_{e}$ as the normalized physical length that a monopole should have to have the first resonance at the same frequency of a given Koch monopole, that is

$$
l_{e}=(4 / 3)^{n} \frac{f_{r}^{0}}{f_{r}^{n}}
$$

where $f_{r}^{0}$ and $f_{r}^{n}$ are the resonant frequencies of the $\mathrm{K} 0$ and $\mathrm{K} n$ antennas, respectively. Fig. 9 shows a plot of the evolution of the electrical length $l_{e}$ as a function of the physical length $l_{p}$ for each new iteration of the Koch monopole. The current distribution shown for the $\mathrm{K} 3$ antenna might suggest that the evolution would be close to $l_{e}=l_{p}$, that is, the electrical length of the antenna increases as the physical length. But, in fact, although the electrical length of the antenna increases at each iteration, it does not grow at the same pace of the physical length. It might even be foreseen as a saturation point, where an increment on the number of fractal iterations does not result in an increment of the electrical size of the antenna. This is probably related to the fact that small details in terms of the wavelength are not relevant to the antenna behavior. Whether this limit value exists, and if it exists, what its value is, does not have a formal 
proof. Even the numerical or empirical determination is difficult. The exponential growth of the number of segments as the fractal iteration increases leads to a huge numerical problem for a relatively small number of iterations. On the other hand, due to a resolution limitation in the manufacturing process there is a technical problem in obtaining Koch antennas with a high number of iterations.

\section{RADIATION PATTERN}

The radiation pattern for the $\mathrm{K} 3$ monopole at the same frequencies of the current distributions of Fig. 8 is shown in Fig. 10. The patterns have been computed as a dipole. The results show that for an antenna length smaller than $0.97 \lambda$, the pattern corresponds to the one of a small antenna, and it is practically the same of the K0 antenna. When the length of the antenna approaches $1 \lambda$, two interesting phenomena appear. First, the null in the $z$ direction disappears. Probably due to the fact that the radiation of the $y$ component of the current does not cancel out anymore. It is also interesting to note that the pattern in the $z y$ plane is asymmetrical. It has to be noted that at this frequency, the overall height of the antenna is $h=0.34 \lambda$, and thus larger than the small antenna limit. Therefore, the phase contribution due to different path-length propagation becomes noticeable in the two abovementioned effects.

\section{THE LONG Koch MONOPOLE}

A final consideration on the possible multiband behavior of the Koch monopole can be made. As shown in [4], the self-similarity properties of certain fractal shapes results in a multiband behavior of the antenna. That is the case of the Sierpinski gasket. In plain words, self-similarity can be described as the replication of the geometry of the structure at a different scale within the same structure. Any fractal defined with an IFS that contains only similarities is self-similar [20]. This is the case of the transformations of (9) that define the Koch curve. On the other hand, an inspection of the geometry of Fig. 2 shows that the lower third of the curve is a replica of the whole curve, but scaled down by one-third. To check the possible multiband behavior of the antenna, the input return loss of the K5 antenna was measured over a wide frequency range. The results shown in Fig. 11 suggest a harmonic behavior rather that a multiband behavior. For comparison, the input return loss of the K0 monopole is also shown. A similar result was found for the Sierpinski gasket monopole with low flare angles [22]. In the case of the Koch monopole, as in the Sierpinski monopoles with low flare angles, the explanation has to be found in the fact that the current distribution is not confined to an active region, but it reaches the end of the antenna, as it was shown in Fig. 8. The fractal structure can be seen as a succession of embedded resonators, each one having the fundamental resonant frequency scaled by a certain factor. This factor is 3 for the Koch curve. The poor radiation resistance of the individual clusters leads to a masking of the log-periodic behavior by the existence of harmonic resonances in the larger clusters of the structure.

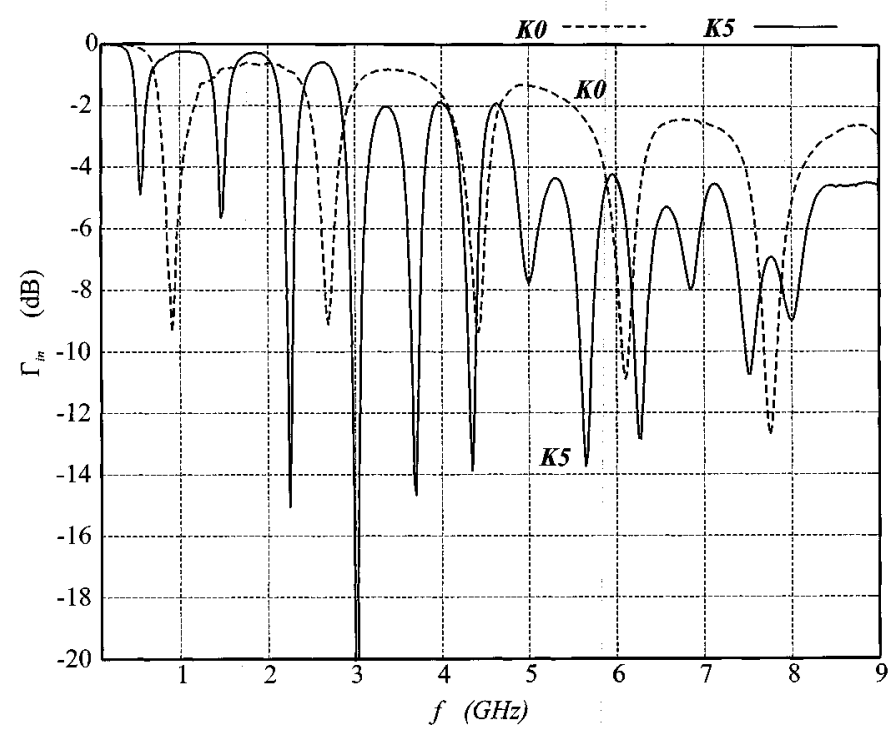

Fig. 11. Measured input return loss of the $\mathrm{K} 5$ and $\mathrm{K} 0$ antennas. The behavior of the input return loss of the K5 antenna suggests a harmonic, rather than a multiband, behavior.

\section{CONCLUSION}

It has been experimentally proven that certain fractal antennas can be very efficient radiators despite their small size in terms of the wavelength. It is well known that there are certain physical constrains to the performance of electrical small antennas. It has been shown that Euclidean-shaped antennas are very far from reaching their limit performance. It has been suggested that this poor behavior is due to the inefficient way that these shapes fill up the volume that encloses them. On the other hand, the fractal counterparts of these antennas having a larger fractal dimension are more efficient in filling up the space. The result is antennas that approach the theoretical limits for small antennas. The practical applications can be readily seen. In a large number of applications, and especially those involving mobile terminals, the reduction of the antenna size is an ultimate goal. The possibility to employ antennas that fit in smaller volumes, but still have an efficient behavior, is certainly appealing. The proof of the existence of a limit in the performance of the antenna as the number of fractal iterations increases is still a challenging research topic.

\section{ACKNOWLEDGMENT}

The authors would like to express their appreciation to FRACTUS for bringing the fractal-shaped antenna concept into practical application.

\section{REFERENCES}

[1] C. Puente, R. Pous, J. Romeu, and X. Garcia, "Antenas fractales o multifractales," Spanish Patent no. 2112163, May 19, 1995.

[2] C. Puente, J. Romeu, R. Pous, X. Garcia, and F. Benitez, "Fractal multiband antenna based on the Sierpinski gasket," Electron. Lett., vol. 32, no. 1, pp. 1-2, Jan. 1996.

[3] C. Puente, "Fractal antennas," Ph.D. dissertation, Dept. Signal Theory and Commun., Universitat Politecnica de Catalunya, June 1997.

[4] C. Puente, J. Romeu, R. Pous, and A. Cardama, "On the behavior of the Sierpinski multiband antenna," IEEE Trans. Antennas Propagat., vol. 46, pp. 517-524, Apr. 1998. 
[5] E. A. Parker and A. N. A. El Sheikh, "Convoluted array elements and reduced size unit cells for frequency-selective surfaces," IEE Proc. $H$ (Microwaves, Optics and Antennas), vol. 138, pp. 19-22, Feb. 1991.

[6] N. Cohen and R. G. Hohfeld, "Fractal loops and the small loop approximation," Commun. Quart., pp. 77-78, Winter 1996.

[7] N. Cohen, "Fractal and shaped dipoles," Commun. Quart., pp. 25-36, Spring 1996.

[8] — "Fractal antennas: Part 2," Commun. Quart., pp. 53-66, Summer 1996.

[9] C. Puente, J. Romeu, R. Pous, J. Ramis, and A. Hijazo, "Small but long Koch fractal monopole," IEE Electron. Lett., vol. 34, pp. 9-10, Jan. 1998.

[10] H. A. Wheeler, "Fundamental limitations of small antennas," Proc. IRE, vol. 35, pp. 1479-1488, Dec. 1947.

[11] R. C. Hansen, "Fundamental limitations on antennas," Proc. IEEE, vol 69, pp. 170-182, Feb. 1981

[12] G. Goubau, "Multielement monopole antennas," in Proc. Workshop on Electrically Small Antennas ECOM, Ft. Monmouth, NJ, May 1976, pp. 63-67.

[13] H. A. Wheeler, "Small antennas," IEEE Trans. Antennas Propagat., vol 23, pp. 462-469, July 1975 .

[14] J. S. McLean, "A re-examination of the fundamental limits on the radiation $Q$ of electrically small antennas," IEEE Trans. Antennas Propagat., vol. 44, pp. 673-676, May 1996.

[15] C. H. Walter, "Electrically small antennas studies at OSU," in Proc. Workshop on Electrically Small Antennas ECOM, Ft. Monmouth, NJ, May 1976, pp. 25-34.

[16] K. Fujimoto, A. Henderson, K. Hirasawa, and J. R. James, Small Antennas. West Sussex: Research Studies Press Ltd., Feb. 1988.

[17] L. J. Chu, "Physical limitations on omni-directional antennas," J. Appl. Phys., vol. 19, pp. 1163-1175, Dec. 1948.

[18] R. E. Collin and C. Rothschild, "Evaluation of antenna Q," IEEE Trans. Antennas Propagat., vol. 12, pp. 23-27, Jan. 1964.

[19] H. O. Peitgen, H. Jurgens, and D. Saupe, Chaos and Fractals, New Frontiers in Science. New York: Springer-Verlag, 1992

[20] J. E. Hutchinson, "Fractals and self-similarity," Indiana Univ. Math. J., vol. 30, pp. 713-747, 1981.

[21] C. A. Balanis, Antenna Theory, Analysis and Design. New York: Wiley, 1982

[22] C. Puente, M. Navarro, J. Romeu, and R. Pous, "Variations on the fracta Sierpinski antenna flare angle," in Proc. IEEE Antennas Propagat. Soc. Int. Symp. Dig., Atlanta, GA, June 1998, pp. 2340-2341.
Carles Puente Baliarda (S'91-M'93) was born in Badalona, Spain, in 1968 $\mathrm{He}$ received the Ingenerio degree in telecommunication engineering from the Polytechnic University of Catalonia (UPC), Barcelona, in 1992, the M.S. degree from the University of Illinois at Urbana-Champaign (UIUC) in 1994, and the Ph.D. degree from the UPC in 1997.

From 1994 to 1999, he joined the faculty of the Electromagnetics and Photonics Engineering group (EEF) at the UPC and worked there in lidar systems and in the development of the fractal technology applied to antennas and microwave devices. He is one of the founders of the company Fractus S.A. and since September 1999, he has held the Technology Director position there. He holds several patents on fractal and other related antenna and microwave inventions.

Dr. Puente Baliarda was awarded with the Best Doctoral Thesis in Mobile Communications 1997 by the Colegio Oficial de Ingenieros de Telecomunicación (COIT) and ERICSSON, and in 1998, he and his team received the European Information Technology Grand Prize from the European Council for the Applied Science and Engineering (EuroCASE) and the European Comission for his work in fractal-shaped antennas and their application to cellular telephony.

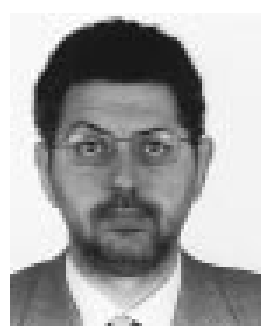

Jordi Romeu (S'88-M'93) was born in Barcelona, Spain, in 1962. He received the Ingeniero and Doctor Ingeniero degrees in Telecommunication Engineering, both from the Polytechnic University of Catalonia (UPC), Barcelona, Spain, in 1986 and 1991, respectively.

In 1985, he joined the Electromagnetic and Photonics Engineering Group of the Signal Theory and Communications Department there. Currently, he is Associate Professor at UPC, where he is engaged in research in antenna near field measurements, antenna diagnostics, and antenna design. He was Visiting Scholar at the Antenna Lab in the University of California, Los Angeles, in 1999 with a NATO Scientific Program scholarship. He holds several patents and has published papers in the fields of antenna near-field measurements and diagnostics, and in antenna design.

Dr. Romeu was Grand Winner of the European IT prize awarded by the European Comission for his contributions in the development of fractal antennas in 1998.

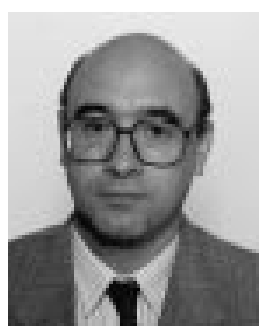

Angel Cardama (S'67-M'73) was born in Santiago de Compostela, Spain, in 1944. He received the Ingeniero de Telecomunicación degree from the Universidad Politécnica de Madrid in 1968 and the Sc.M. and Ph.D. degrees in electrical engineering from Brown University, Providence, RI, in 1970 and 1973, respectively.

In 1972, he joined the faculty of the Telecommunication Engineering School at the Polytechnic University of Catalonia, Barcelona, Spain, where he holds the position of Professor. His research interests range from the development of analytical and numerical techniques in electromagnetics to the design of microwave imaging systems and radar and communications antennas. 